\title{
Breaking Bad: Towards Certifiable Additively Manufactured Alloys Using Post-Build Heat Treatment
}

\author{
Fan Zhang ${ }^{1}$, Carelyn E. Campbell ${ }^{1}$, Mark R. Stoudt ${ }^{1}$, Lyle E. Levine ${ }^{1}$, Andrew J. Allen ${ }^{1}$, Eric A. Lass ${ }^{2}$, and \\ Greta Lindwall ${ }^{3}$ \\ ${ }^{I}$ Material Measurement Laboratory, National Institute of Standards and Technology, Gaithersburg, MD 20899, USA. \\ ${ }^{2}$ Department of Materials Science and Engineering, University of Tennessee, Knoxville, TN 37996, USA. \\ ${ }^{3}$ Department of Materials Science and Engineering, KTH Royal Institute of Technology, Brinellvägen 8, 11428 Stockholm, Sweden
}

fan.zhang@nist.gov

Additive manufacturing (AM) of metals provides great flexibility in manufacturing parts with complex geometrical shapes and is fast becoming an attractive option for the fabrication of high-valued metal components in aerospace, oil \& gas, and biomedical industries. The rapid heating and cooling during AM fabrication, which by nature is a highly nonequilibrium process, often leads to significant microstructural heterogeneity uncommon to wrought and cast alloys. Such heterogeneity creates tremendous challenge in the qualification and eventual certification of AM metal parts for many applications.

Using a combination of in situ synchrotron-based X-ray scattering and diffraction methods, ex situ electron microscopy, atom-probe tomography, and thermokinetic and thermodynamic modelling, we have focused on the development of post-build heat treatment protocols for AM alloys. Our established protocols recover the designed phase composition of two types of widely used commercial AM alloys, a major step towards their part certification. Specifically, our work on AM nickel-based superalloy Inconel 625 demonstrates the importance of understanding the effect of elemental microsegregation, a ubiquitous phenomenon in AM alloys resulting from rapid solidification, on the structure and microstructure evolution during post-build heat treatments [1]. Our simulationconstructed and experiment-validated time-temperature-transformation diagram clearly demonstrates the acceleration (by a factor of $100-1000$ ) of formation kinetics of a phase deleterious to the fatigue performance of this alloy $[2,3]$. Our work on nitrogen-atomized 17-4 stainless steel shows that the starting powder chemistry and compositional partition during solidification results in the asfabricated 17-4 being fully austenitic, as opposed to being fully martensitic as designed. Our three-step heat treatment protocol successfully recovers the martensitic structure of parts fabricated using nitrogen-atomized 17-4 powders [4]. We also determined the optimal ageing heat treatment to yield optimal strength of this precipitation-hardening alloy.

Our work points to a common and important theme that post-build heat treatment is critical for producing AM alloys with predictable and reproducible microstructures and hence materials properties. The emphasis of proper post-build heat treatment cannot be overstated for the certification of many AM alloys. We also emphasize that rigorous and in situ bulk structure and microstructure measurements only available at synchrotrons are essential for modelers to validate AM simulations for the advancement of AM technologies [5].

[1] Zhang, F., Levine, L. E., Allen, A. J., Stoudt, M. R., Lindwall, G., Lass, E. A., Williams, M. E., Idell, Y. \& Campbell, C. E. (2018). Acta Materialia 152, 200-214.

[2] Stoudt, M. R., Lass, E., Ng, D. S., Williams, M. E., Zhang, F., Campbell, C. E., Lindwall, G. \& Levine, L. E. (2018). Metallu rgical and Materials Transactions A 49, 3028-3037.

[3] Lindwall, G., Campbell, C., Lass, E., Zhang, F., Stoudt, M. R., Allen, A. J. \& Levine, L. E. (2019). Metallurgical and Materials Transactions A 50, 457-467.

[4] Lass, E. A., Zhang, F. \& Campbell, C. E. (2020). Metallurgical and Materials Transactions A, 1-15.

[5] Zhang, F., Levine, L. E., Allen, A. J., Young, S. W., Williams, M. E., Stoudt, M. R., Moon, K.-W., Heigel, J. C. \& Ilavsky, J. (2019). Integrating Materials and Manufacturing Innovation 8, 362-377

Keywords: Additive manufacturing; metals and alloys; structure; microstructure; in situ characterization; synchrotron; SAXS

Portions of this research were performed on beamline 9-ID-C, 11-ID-B, and 11-BM at the Advanced Photon Source, a U.S. Department of Energy (DOE) Office of Science User Facility operated for the DOE Office of Science by Argonne National Laboratory under Contract No. DE-AC02-06CH11357. 